\title{
Correspondence: T cells are compromised in tetracycline transactivator transgenic mice
}

\author{
Anja Schmitt ${ }^{1}$ Klaus Schulze-Osthoff ${ }^{1,2} \cdot$ Stephan Hailfinger $^{1}$
}

Received: 4 November 2017 / Revised: 17 November 2017 / Accepted: 20 November 2017 / Published online: 19 January 2018

(c) ADMC Associazione Differenziamento e Morte Cellulare 2018

Tetracycline (Tet)-controlled transcriptional activators allow for the inducible expression of protein-coding genes or shRNAs, and are frequently used to decipher gene function in cultured cells and in vivo [1]. The system is based on the bacterial Tet operon that mediates resistance to Tet derivatives, such as doxycycline (Dox). In the Tet-off system, fusion of a Tet repressor protein with the transcriptional activation domain of the herpes simplex virus protein VP16 results in a Tet-controlled transcriptional activator (tTA), which constitutively binds to Tet-responsive elements (TRE), but is inhibited in its transcriptional activity by Dox [1]. In contrast, in the more frequently used Tet-on system, a mutant tTA-version generates a reverse tTA (rtTA) that is only recruited to the TRE upon Dox binding and then mediates transgene expression [1]. Since the Tet-system allows transgene expression in a tissue-specific and temporal manner, it has been extensively used for regulating expression of oncogenes or silencing of tumor suppressor genes [1-3]. Despite its enormous success, however, we report here that, irrespective of the transgene, the Tet-system might compromise activated T-cells, thereby providing a cautionary note for its use in immunological studies.

To investigate T-cell responses, we used transgenic mice carrying an advanced Tet-on transactivator, driving

Electronic supplementary material The online version of this article (https://doi.org/10.1038/s41418-017-0042-y) contains supplementary material, which is available to authorized users.

Klaus Schulze-Osthoff

kso@uni-tuebingen.de

Stephan Hailfinger

stephan.hailfinger@uni-tuebingen.de

1 Department of Molecular Medicine, Interfaculty Institute for Biochemistry, University of Tübingen, 72076 Tübingen, Germany

2 German Cancer Consortium (DKTK) and German Cancer Research Center (DKFZ), 69120 Heidelberg, Germany expression of a miR30-based shRNA in frame with a turboGFP reporter. To reduce effects associated with random integration, the Dox-inducible shRNA-reporter transgene was delivered by a recombinase-mediated cassette exchange approach into the type-I collagen (Collal) locus. During an in-depth analysis of the transgenic mice, we discovered that specific splenic T-cell populations were absent not only in those mice expressing shRNAs targeting particular mRNAs, but surprisingly also in mice carrying a control shRNA for Renilla luciferase. These effects were independent of the promoter for rtTA expression, and observed in independent mouse lineages of both ROSA26 and CAG promoter-driven systems. Although total $\mathrm{CD} 4^{+} \mathrm{T}$-cells in the spleen were only marginally reduced, antigen-experienced $\mathrm{CD} 4^{+} \mathrm{T}$ cells, which we characterized as $\mathrm{CD} 44^{+}$and $\mathrm{CD} 62 \mathrm{~L}^{\text {low }}$, were nearly absent in the GFP-positive fractions (Fig. 1). We confirmed the reduction of activated T-cells in the GFPpositive population by CD25 and CD69 staining (Fig. 1). Importantly, disappearance of antigen-experienced T-cells was not mediated by Dox itself, since Dox-treated mice lacking rtTA expression exhibited normal numbers of activated T-cells (Fig. 1). Already 6-10 days of Dox treatment were sufficient to trigger a profound reduction in $\mathrm{CD} 25^{+} / \mathrm{CD} 44^{+} / \mathrm{CD} 4^{+} \mathrm{T}$-cells, which characterize effector memory or regulatory T-cells. Thus, their rapid disappearance suggests that Dox-activated rtTA/turboGFP expression induces toxicity in these cells (Fig. 1). Interestingly, the frequency of double-negative thymocytes expressing CD25 and CD44 was not altered (Suppl. Fig. S1). However, antigen-experienced GFP-positive CD8 ${ }^{+}$T-cells, characterized by CD44 expression, were reduced similarly to the $\mathrm{CD} 4^{+}$lineage (Suppl. Fig. S2).

Our findings demonstrate that, in contrast to thymocytes and naïve T-cells, antigen-experienced T-cells are depleted using the rtTA-system. Hundreds of mouse models have been generated with the rtTA-system for the expression of oncogenes or the silencing of tumor suppressor genes. In the latter condition, the blunting effects on T-cell activation might be even advantageous to identifying novel tumor 
suppressors. More recently, Tet-transactivators proved very useful for modulating gene activities by CRISPR/Cas9 and Cre/LoxP technologies [4, 5]. Adverse effects of the rtTA- protein might not be restricted to activated T-cells, but could also affect other cell populations [6, 7]. Importantly, a recent study provided compelling evidence that not only
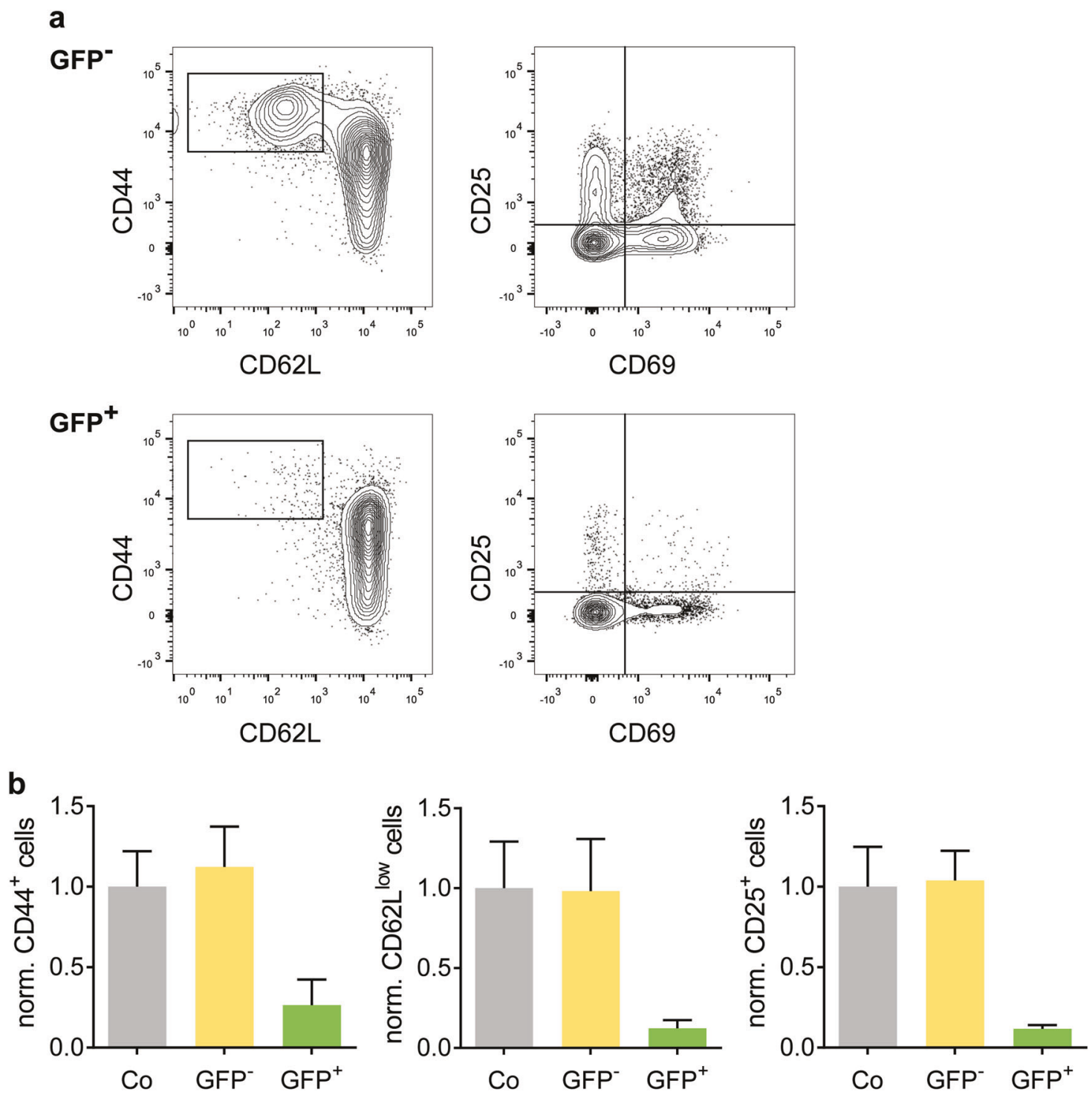

C
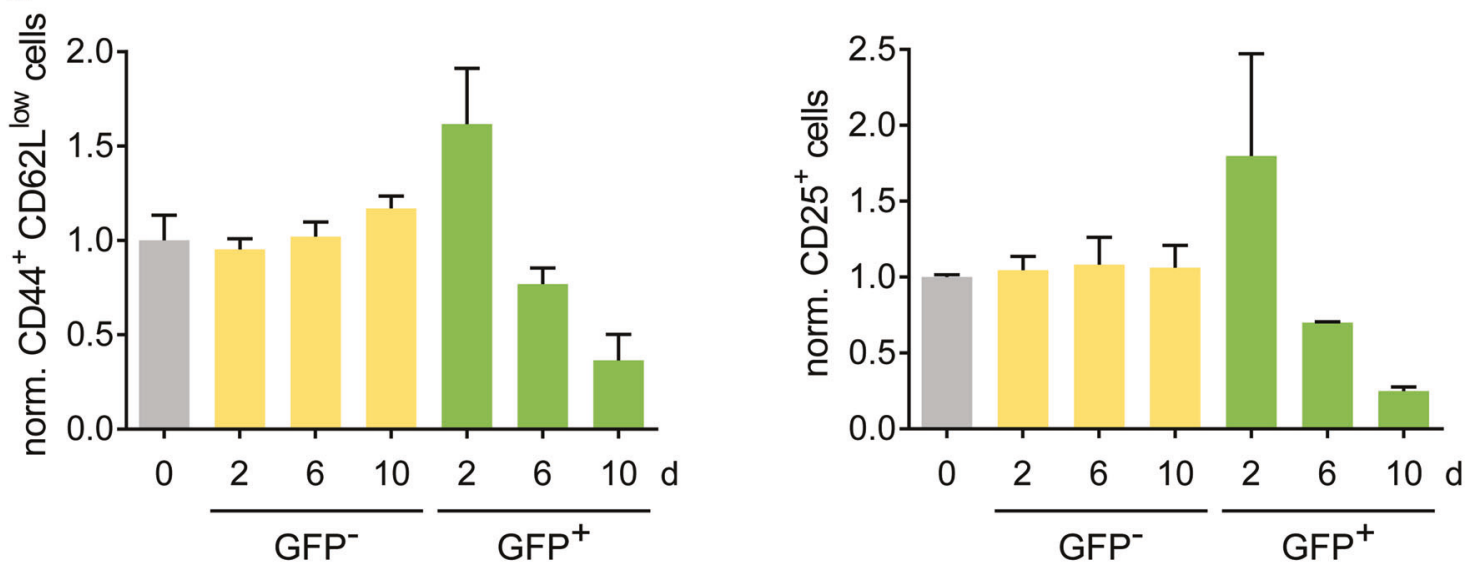
Fig. 1 Quantification of antigen-experienced splenic T-cells in ROSA26-rtTA TRE-turboGFP-shRenilla mice. a Flow cytometric analysis of total splenocytes isolated from mice receiving $2 \mathrm{mg} / \mathrm{ml}$ doxycycline (Dox) for 4 weeks. Splenocytes were gated for CD4 ${ }^{+}$ cells and segregated by their GFP intensity in two populations (GFP and $\mathrm{GFP}^{+}$). Antigen-experienced $\mathrm{CD}^{+}{ }^{+} \mathrm{T}$-cells were quantified by the combination of $\mathrm{CD} 44^{+}$and $\mathrm{CD} 62 \mathrm{~L}^{\text {low }}$ or by the cell surface expression of the activation markers CD25 and CD69. b The frequency of $\mathrm{CD} 44^{+}, \mathrm{CD} 62 \mathrm{~L}^{\text {low }}$, and $\mathrm{CD} 25^{+}$splenic $\mathrm{CD} 4^{+} \mathrm{T}$-cells from ROSA26rtTA TRE-turboGFP-shRenilla mice was normalized to control mice lacking rtTA expression. Following Dox treatment for four weeks, at least six mice were analyzed per group. c Quantification of antigenexperienced splenic $\mathrm{CD}^{+}{ }^{+} \mathrm{T}$-cells during short-term Dox treatment. After 2, 6, and 10 days, the frequency of splenic $\mathrm{CD} 4^{+} \mathrm{T}$-cells expressing $\mathrm{CD}_{4} 4^{+}, \mathrm{CD} 62 \mathrm{~L}^{\text {low }}$, and $\mathrm{CD} 25^{+}$was determined in the $\mathrm{GFP}^{+}$and $\mathrm{GFP}^{-}$population and normalized to untreated ROSA26rtTA TRE-turboGFP-shRenilla mice

activated T-cells, but also B-cells are diminished in tTAtransgenic mice [8]. This study further showed an inhibition of lymphocyte depletion by Bcl-2, suggesting that tTA expression triggers an apoptotic response. Thus, in addition to the ability of Dox to interfere with mitochondrial functions [9], these results provide a cautionary note for using proper controls to reveal potential side effects of Tet transactivator expression alone.

Acknowledgements We thank A. Villunger for sharing data prior to publication and S. Autenrieth for helpful discussion. This work was supported by the Emmy-Noether Program, the CRC TR156 and TR209 of the German Research Foundation, and the Excellence Initiative of the University of Tübingen.

\section{Compliance with Ethical Standards}

Conflict of interest The authors declare that they have no conflict of interest.

\section{References}

1. Gossen M, Bujard H. Annu Rev Genet. 2002;36:153-73.

2. Welman A, et al. Transl Oncogenomics. 2007;2:17-33.

3. Premsrirut PK, et al. Cell. 2011;145:145-58.

4. Schonig K, et al. Nucleic Acids Res. 2002;30:e134.

5. Dow LE, et al. Nat Biotechnol. 2015;33:390-4.

6. Sisson TH, et al. Am J Respir Cell Mol Biol. 2006;34:552-60.

7. Morimoto M, et al. Dev Biol. 2009;325:171-8.

8. Ottina E, et al. Nat Commun. 2017;8:1028.

9. Moullan N, et al. Cell Rep. 2015;10:1681-91 